\title{
Estimating the Global Burden of Foodborne DISEASES - A COLLABORATIVE EFFORT
}

\author{
T Kuchenmüller (kuchenmullert@who.int) ${ }^{1}$, S Hird ${ }^{1}$, C Stein ${ }^{1}$, P Kramarz ${ }^{2}$, A Nanda ${ }^{2}$, A H Havelaar ${ }^{3}$ \\ 1. Department of Food Safety, Zoonoses and Foodborne Diseases, World Health Organization, Geneva, Switzerland \\ 2. European Centre for Disease Prevention and Control, Stockholm, Sweden \\ 3. Laboratory for Zoonoses and Environmental Microbiology, National Institute for Public Health and the Environment, Bilthoven, \\ the Netherlands
}

IIIness and death from diseases caused by unsafe food are a constant threat to public health security as well as socioeconomic development throughout the world. The full extent of the burden and cost of foodborne diseases associated with pathogenic bacterial, viral and parasitic microorganisms, and food contaminated by chemicals is still unknown but is thought to be substantial. The World Health Organization (WHO) Initiative to estimate the global burden of foodborne diseases aims to fill the current data gap and respond to the increasing global interest in health information. Collaborative efforts are required to achieve the ambitious task of assessing the foodborne disease burden from all causes worldwide. Recognising the need to join forces, the WHO Initiative has assembled an alliance of stakeholders which share and support the Initiative's vision, intended objectives and outcomes. One important collaborator is the European Centre for Disease Prevention and Control (ECDC) which has embarked on a burden of disease study covering at least 18 foodborne diseases in nearly 30 countries.

\section{Burden of foodborne diseases}

All countries have limited resources with which to address the health needs of their populations. Decision makers therefore need access to high-quality scientific evidence in order to prioritise resource allocation and improve public health in the most efficient and effective manner possible [1].

Surveillance data are often considered as one of the main evidence bases underpinning public health policy decisions. However traditional surveillance systems tend to capture merely a fraction of the existing disease burden. For data on foodborne diseases to be included, the affected persons need to seek medical care, provide a specimen, and test positive on laboratory tests. Moreover, the results have to be reported to the relevant health authorities [2]. The spectrum of pathogens causing infectious diseases is vast, and the diversity of these diseases makes it difficult to use surveillance data to set priorities to enable the best use of resources [3]. In addition, there are few surveillance systems which capture and attribute human illness due to infections following the ingestion of specific foods or sequelae that may be associated with foodborne infections, such as Guillain-Barré syndrome following campylobacteriosis, or epilepsy associated with neurocysticercosis following infection with the parasite Taenia solium.
Using the burden of disease methodology enables public health officials to circumvent some of the problems posed by the difficulty to report properly the incidence of foodborne diseases. 'Burden of disease' has been defined as the incidence and/or prevalence of morbidity, disability, and mortality associated with acute and chronic manifestations of disease [4]. The overall burden of disease is estimated using various composite measures of population health status such as the disability-adjusted life year (DALY), which is a time-based measure that combines years of life lost due to premature mortality and years of life lost due to time lived in disability or states of less than full health [5].

The burden of disease metric has been used extensively by the World Health Organization (WHO) and others to describe the global, regional and national burden from diseases [5]. Although some countries have recently quantified the national burden of foodborne diseases $[6,7]$ the overall burden of these diseases has not been fully described to date.

Why estimate the global burden of foodborne diseases?

Through the globalisation of food marketing and distribution, both accidentally and deliberately contaminated food products can affect the health of people in numerous countries at the same time. This has been demonstrated by recent events surrounding melamine contamination in food [8]. Moreover, foodborne diseases appear to be emerging more frequently than ever before and the capacity of public health authorities to apply conventional control measures does not seem to be developing at the same speed [9]. A recent publication in Nature has shown that approximately $30 \%$ of all emerging infections over the past 60 years were caused by pathogens commonly transmitted through food [10]. This trend is compounded by the growing industrialisation of food and feed production as well as intensive farming which catalyses the appearance and spread of pathogens (e.g. prions associated with Bovine spongiform encephalopathy (BSE) leading to new variant Creutzfeldt-Jakob disease (VCJD) in humans during the 1990s which was caused by the use of meat and bone meal in the production of animal feeds [11]).

Diarrhoeal diseases alone - a considerable proportion of which is foodborne - kill 2.2 million people globally every year [12], but the burden arising from all foodborne diseases is clearly larger. 
The heaviest share of the disease burden occurs in poor countries and jeopardises international development efforts, including the achievement of the Millennium Development Goals (MDGs). The MDG's are eight specific development goals that aim to combat extreme poverty around the world, to be met by 2015 and that were endorsed at the UN Millennium Summit in 2000 [13]. Indeed, several analyses have shown that to attain MDG 4 which focuses on reducing the under-five mortality rate by two thirds between 1990 and 2015, renewed efforts are needed to prevent and control diarrhoea, among other diseases [12].

In order to generate data on the full extent and cost of foodborne diseases, the WHO Department of Food Safety, Zoonoses and Foodborne Diseases (FOS) launched the Initiative during an international consultation in 2006 [4]. The Initiative aims to provide the first ever quantitative description of foodborne disease burden by 2011, when estimates of the burden of foodborne diseases worldwide will be generated according to age, sex and WHO regions for a defined list of causative agents of microbial, parasitic, and chemical origin. This information will enable policymakers and others to:

- appropriately allocate resources to foodborne disease, prevention and control efforts;

- monitor and evaluate food safety measures;

- develop new food safety standards;

- assess the cost-effectiveness of interventions;

- quantify the burden in monetary costs, and

- attribute human illness to specific food sources to support risk management strategies [2].

Foodborne Disease Burden Epidemiology Reference Group (FERG) - an external expert group advising WHO

One of the main recommendations of the 2006 consultation was to establish a Foodborne Disease Burden Epidemiology Reference
Group (FERG) which would advise the WHO on the generation of comprehensive foodborne disease burden estimates. The principles behind the FERG are based on a detailed analysis of lessons learnt from other external WHO expert groups, such as the Monitoring and Evaluation Reference Group (MERG) for malaria or the Child Health Epidemiology Reference Group (CHERG) [14].

The FERG is a group which unites disciplines that do not traditionally tend to collaborate, such as: risk assessment and epidemiology, microbiology, virology, parasitology, toxicology and disease and exposure modelling. This multidisciplinary approach enables the group to generate comprehensive data from all major foodborne diseases. The FERG is mandated to:

- assemble, appraise and report on existing burden of foodborne disease estimates

- conduct epidemiological reviews of mortality, morbidity and disability for each of the major foodborne diseases as determined by the FERG (for more details see the meeting report, [9]);

- provide models for the estimation of foodborne disease burden where data are lacking;

- develop cause and source attribution models to estimate the proportion of diseases that are foodborne, and

- develop user-friendly tools for foodborne disease burden studies at country level.

The FERG operates through a Core Group, five Task Forces, and ad hoc Resource Advisers.

The WHO Secretariat carries out logistic, administrative, and technical support functions (Figure).

Since its establishment, the FERG has met twice to (a) decide on priority causative agents for which burden data should be generated (for more details see the meeting report, [9]), (b)

F I G U R E

Composition and structure of the WHO Initiative to Estimate the Global Burden of Foodborne Diseases [9]

The FERG Core or Steering Group functions as steering committee and consists of scientists from each of the areas outlined in the Task Forces below. It is charged with monitoring and appraising the technical and epidemiological work of all the Task Forces and is chaired by a scientist with extensive international experience in both foodborne diseases and burden of disease methodology.

\section{WHO Secretariat}

The WHO Secretariat is composed of staff from eight WHO Departments having a stake in foodborne diseases andior burden of disease, as well staff from UN partner organizations.

Thematic Task Force 1:

advancing the burden work in the area of viral and bacterial enteric diseases.

Thematic Task Force 2: advancing the burden work in the area of parasitic diseases.

Thematic Task Force 3:

advancing the burden work in the area of chemicals and toxins.

Thematic Task Force 4

on source attribution (charged with identifying the proportion of disease burden that is directly due to food contamination and aiming to attribute the relevant fraction of disease burden to the specific food source responsible).

Thematic Task Force 5:

FERG Resource Advisers:
FERG ad hoc

Resource

Advisers
Additional external experts can be called upon to join the FERG on an ad-hoc basis to supplement the skills required. on country studies (which will be set up in June 2009 to develop user-friendly tools for countries to conduct their own burden of foodborne disease studies, thus enabling them to monitor the pro gress of their food safety interventions). 
develop extensive workplans guiding the WHO Secretariat on the work to be commissioned, and (c) appraise the progress made with commissioned work. Major pieces of review, research and modelling work have been undertaken by externally commissioned scientists for the following causative agents:

- chemicals/toxins: cyanide from cassava, aflatoxin, dioxins, peanut allergens;

- parasites: intestinal protozoa, Fasciola hepatica, Taenia solium, Echinococcus multilocularis;

- enteric pathogens: global burden of diarrhoeal diseases in persons older than five years of age.

First interim results are expected in 2009. A peer-review system involving external reviewers increases the quality and scientific rigour of the work of the FERG.

The Task Force on Source Attribution (task force 4), aiming to attribute the relevant fraction of disease burden to the specific food source responsible, commenced its work in April 2008. The fifth FERG Task Force on country studies will commence its work in June 2009. This task force will increase the capacity of countries to conduct their own foodborne disease burden assessments. Eighteen country studies are envisaged (three in each of the six WHO regions), and will provide first-hand field data, fill data gaps identified by the FERG, and help validate the burden results generated by modelling approaches.

\section{Partnerships - joining efforts for results}

The multifactorial nature of foodborne diseases necessitates close collaboration between the WHO Initiative and a large number of partners and stakeholders, to bring together necessary expertise and resources, and minimise duplication of efforts. The Initiative is capitalising on existing WHO in-house experience with staff from several WHO departments dealing with diseases of potentially foodborne origin (including child health, parasitic and neglected tropical diseases, water and sanitation, and others), working with the Initiative.

\section{Collaboration with external stakeholders}

The Initiative relies on an alliance of external collaborators and partners who provide technical expertise, information sharing platforms, networking possibilities and/or financial support. Through the FERG members, more than 30 internationally renowned scientific institutions from all over the world have been linked with the Initiative. WHO has established close technical collaboration with several organisations involved in major global and regional burden of disease initiatives, including (among others):

- The European Centre for Disease Prevention and Control (ECDC) which has embarked on a burden of disease study covering nearly 30 countries and up to 49 infectious diseases, of which at least 18 can also be transmitted by food (see also the section below on collaboration with the ECDC).

- The Institute for Health Metrics and Evaluation (IHME) in Seattle which is updating the Global Burden of Disease data for the year 2005, the year of reference. The risk factor 'unsafe food' will not be examined by IHME, but will instead be assessed by the WHO Initiative due to its specific knowledge in this area.

- The International Collaboration on Enteric Disease Burden of IIIness Studies which facilitates communication between experts who have conducted burden of enteric or foodborne infectious disease studies.
- Med-Vet-Net, a European research network for zoonoses, which will produce estimates of the disease burden and cost of illness of (selected) foodborne and zoonotic pathogens in eight European countries.

The WHO has assembled and continues to expand an alliance of funding agencies and in kind supporters for the FERG, to ensure that no individual agency, foundation, or government can exert undue influence on the Initiative. The WHO and other institutions (such as the Ministry of Health, Welfare and Sport, the Netherlands; the Centers for Disease Control and Prevention and the United States Department of Agriculture, United States; the Ministry of Health, Labour and Welfare, Japan; the Department of Health, United Kingdom) continue to make considerable financial investments in the Initiative. The WHO is currently discussing additional funding options with a number of governmental and non-governmental donors.

\section{Stakeholder events}

The Initiative has implemented a detailed communication strategy covering internal and external information sharing, mechanisms for accountability, as well as all aspects of advocacy. Key food safety stakeholders were invited to the first formal meeting of the FERG in November 2007 to give their input to the Initiative. This involvement proved to be very fruitful, and the input received from the stakeholders was endorsed in the technical deliberations of the FERG [9].

The second FERG meeting (17 to 21 November 2008) also incorporated a stakeholder gathering. Representatives from more than 30 institutions (including the WHO Member States, bi- and multilateral organisations, agricultural and food industry, consumer groups, academia as well as scientific and public media) attended the event in November 2008. Stakeholders welcomed the WHO's effort to estimate the foodborne disease burden.

Working group sessions at the meeting provided an opportunity for all participants to interact directly with the Initiative and the FERG members and to give relevant suggestions in the areas of communications, advocacy and policy [15].

\section{Collaboration with the ECDC}

The WHO has a global mandate to assemble health information, assist countries to shape the health research agenda, set norms and standards, monitor and assess health trends and provide technical support to countries. The ECDC is responsible for identifying, assessing and communicating current and emerging threats to human health from infectious diseases within the European Union (EU) [16]. The WHO and the ECDC work closely together in order to avoid duplicating efforts and to make the best use of limited resources.

In 2006 the ECDC recognised that a composite measure of disease burden, such as DALY, could be used to guide public health policy and action in the area of communicable diseases [17]. Therefore a three-month pilot study to explore the potential of the disease burden concept for seven communicable diseases was conducted [18].

A study called "Present and Future Burden of Communicable Diseases in Europe" (BCoDE) will build on the pilot results, and will make use of existing methodologies such as those developed by the 
WHO for its Global Burden of Disease Study [19]. The ECDC project is planned to start in 2009 with the initial phase (methodology development, field testing and full burden study) estimated to last four years. The burden of disease estimates will subsequently be updated on a regular basis.

While there is some overlap between the two studies with regards to the diseases (about one third of the diseases covered in the EU-wide study involving foodborne pathogens are also being investigated by the FERG), the effort of the WHO Initiative focuses on the global picture of all major foodborne diseases, including those resulting from chemical and numerous parasitic hazards which are not covered by the ECDC's study. Additionally, the FERG aims to attribute causes of disease burden to particular food commodities, where possible. To ensure a synergistic approach, scientists from the ECDC and all relevant networks are represented as advisers on the FERG.

\section{Conclusions}

Assessing the global burden of foodborne diseases from all major causes using summary health metrics in the form of the DALY is needed to help decision makers allocate appropriate resources to food safety control and prevention. To tackle this large task, the Initiative to Estimate the Global Burden of Foodborne Diseases combines the WHO's public health leadership capacity with the independent expert advice of FERG, and relies on an inter-sectoral alliance of partners and stakeholders.

Multi-stakeholder partnerships work best if aligned with the strategic interests of each party. This is the case for the ECDC and the WHO Initiative. Both institutions aim to estimate the burden of foodborne diseases by capitalising on their respective strengths. The ECDC will generate burden data on communicable diseases (including those transmitted by food) for European countries whereas the WHO will focus on the global burden of foodborne diseases from all major causes. Based on complementary strengths, this process will enable both institutions to avoid duplication of efforts, share technical expertise and data, as well as ensure comparability of burden results.

The WHO Initiative is continuously seeking to broaden its cooperation with external partners. The annual stakeholder meetings have proven to be an effective platform for fostering constructive dialogue and interaction between the WHO, the FERG and the food safety stakeholder community. These meetings will increase in size and importance to further catalyse international collaboration and funding for effective foodborne diseases prevention and intervention measures.

\section{Authors' disclaimer}

The findings and conclusions in this publication are those of the authors and do not necessarily represent the decisions or policies of their respective institutions.

\section{Aknowledgements}

The authors wish to thank J Takkinen and T Westrell, Surveillance Unit, European Centre for Disease Prevention and Control.

\section{References}

1. World Health Organization. The evidence-informed policy network (EVIPNet). Available from: http://evipnet.bvsalud.org/php/index.php?lang=en
2. World Health Organization. Initiative to Estimate the Global Burden of Foodborne Diseases. Available from: http://www.who.int/foodsafety/foodborne disease/ferg/en/index.html

3. Krause G, Working Group on Prioritisation at Robert Koch Institute. Prioritisation of infectious diseases in public health - call for comments. EuroSurveill. 2008;13(40):pii=18996. Available from: http://www. eurosurveillance.org/images/dynamic/EE/V13N40/art18996.pdf

4. World Health Organization. WHO consultation to develop a strategy to estimate the global burden of foodborne diseases. Geneva: World Health Organization; 2006. p. vii. Available from: http://www.who.int/foodsafety/publications/ foodborne_disease/fbd_2006.pdf

5. Murray CJL, Lopez AD, editors. The global burden of disease: a comprehensive assessment of mortality and disability from diseases, injuries, and risk factors in 1990 and projected to 2020. Cambridge (MA): Harvard University Press; 1996.

6. Kreijl CF van, Knaap AGAC, Raaij JMA van, editors, Our food, our health. Healthy diet and safe food in the Netherlands. Bilthoven: RIVM; 2006. Available from: http://www.rivm.nl/bibliotheek/rapporten/270555009.pdf

7. World Health Organization. WHO consultation to develop a strategy to estimate the global burden of foodborne diseases. Geneva, 2006. p. 16-17. Available from: http://www.who.int/foodsafety/publications/foodborne_disease/ fbd_2006.pdf

8. World Health Organization. Melamine-contamination event. Available from: http://www.who.int/foodsafety/fs_management/infosan_events/en/index.html

9. World Health Organization. WHO Initiative to Estimate the Global Burden of Foodborne Diseases. First formal meeting of the Foodborne Disease Burden Epidemiology Reference Group. Geneva, 2007. Available from: http://www.who. int/foodsafety/publications/foodborne_disease/burden_nov07/en/index.html

10. Jones KE, Patel NG, Levy MA, Storeygard A, Balk D, Gittleman JL, et al. Global trends in emerging infectious diseases. Nature. 2008;451(7181):990-3.

11. Smith PG, Bradley R. Bovine spongiform encephalopathy (BSE) and its epidemiology. Br Med Bull. 2003;66(1):185-98.

12. World Health Organization. The Global Burden of Disease. 2004 Update. Geneva, 2008. Available from: http://www.who.int/healthinfo/global_burden_disease/ GBD_report_2004update_full.pdf

13. United Nations. End Poverty 2015. Millennium Development Goals. Available from: http://www.un.org/millenniumgoals/

14. Stein C, Kuchenmüller T, Hendrickx S, Prüss-Üstün A, Wolfson L, Engels $D$, et al. The global burden of disease assessments - WHO is responsible? Negl Trop Dis. 2007;1(3):1-8

15. World Health Organization. WHO Initiative to Estimate the Global Burden of Foodborne Diseases. Increasing impact through collaboration. Foodborne Disease Stakeholder Meeting, 20 November 2008, Geneva. Geneva, 2009. Available from: http://www.who.int/foodsafety/foodborne_disease/ferg1/en/ index.html

16. European Centre for Disease Prevention and Control (ECDC) [homepage on the internet]. Available from: http://ecdc.europa.eu/en/About_us/Default.aspx

17. Jakab Z. Why a burden of disease study?. Euro Surveill. 2007;12(12):pij=750. Available from: http://www.eurosurveillance.org/ViewArticle. aspx?ArticleId $=750$

18. van Lier EA, Havelaar AH, Nanda A. The burden of infectious diseases in Europe a pilot study, Euro Surveill, 2007:12(12):pij=751. Available from: http://www. eurosurveillance.org/ViewArticle.aspx?ArticleId $=751$

19. World Health Organization. Global Burden of Disease project. Available from: http://www.who.int/topics/global_burden_of_disease/en/

This article was published on 7 May 2009.

Citation style for this article: Kuchenmüller T, Hird S, Stein C, Kramarz P, Nanda A Havelaar AH. Estimating the Global Burden of Foodborne Diseases - a collaborative effort. Euro Surveill. 2009;14(18):pii=19195. Available online: http://www.eurosurveillance.org/ ViewArticle.aspx?ArticleId $=19195$ 